\title{
Os desafios da educação permanente: a experiência do curso de Medicina da Universidade Estadual de Londrina
}

\author{
The challenge of faculty development: the \\ experience of the medical school at the State \\ University of Londrina
}

\author{
Maria José Sparça Salles de Faria ${ }^{1}$ \\ Elisabete de Fátima Polo de Almeida Nunes ${ }^{1}$ \\ Lea Anastasiou ${ }^{2}$ \\ Marcia Hiromi Sakai ${ }^{1}$ \\ Vera Lúcia Menezes da Silva ${ }^{1}$
}

\footnotetext{
PALAVRAS-CHAVE

- Educação Médica.

- Apoio ao Desenvolvimento de Recursos Humanos.

- Corpo Docente.

- Aprendizagem Baseada em Problemas.
}

\section{KEY WORDS \\ - Medical Education. \\ - Staff Development. \\ - Faculty, Medical. \\ - Problem-Based Learning.}

Recebido em: 10/07/2007

Reencaminhado em: 10/01/2008

Aprovado em: 10/02/2008

REVISTA BRASILEIRA DE EDUCAÇ̃̃o MÉDICA

\footnotetext{
${ }^{1}$ Universidade Estadual de Londrina, Paraná, Brasil.

2 Universidade Estadual de Londrina, Paraná, Brasil; Universidade de São Paulo, São Paulo, Brasil.
}

Faculty staff of undergraduate medical school has usually a high level of techno-scientific knowledge but deficient didactic and pedagogical competences, resulting in resistance to change. The purpose of this article is to discuss the challenges posed to the implementation of a faculty development program for the medical course of the State University of Londrina. The faculty development committee introduced two activities, as there are: a) An introductory course to prepare future tutors who will provide support to the tutorial group using problem-based learning methods; b) The establishment of a study group in order to discuss and reflect about topics related to the teaching practice.

For overcoming the challenges posed by nurturing medical faculty development all implanted activities need to be constantly evaluated in order to assure continuing capacity building. 


\section{INTRODUÇÃO}

A sociedade atual vivencia a velocidade da mudança, impulsionada pela aceleração da produção, aplicação e difusão do conhecimento científico e sua concretização em processos e produtos tecnológicos que invadem o cotidiano individual e coletivo. Ocorre ainda uma globalização competitiva, que gera a multiplicação de processos de exclusão. Se não compete à escola responder por essas mudanças sociais significativas, cabe a ela questionar os fins e a função da universidade neste contexto, mediante a natureza da educação oferecida a todos, como direito de cidadania, e a função social que cabe à educação superior ${ }^{1,2,3}$.

Para isso, a ação de educadores deve atuar na superação de uma comunicação de massa, linear e impositiva, na direção de uma comunicação interativa, em que se estabeleça uma relação recíproca, dialógica e democrática ${ }^{3,4}$.

A Universidade Estadual de Londrina (UEL) adotou como uma das estratégias de ensino a Aprendizagem Baseada em Problemas $(\mathrm{ABP})^{5}$ a partir de 1998. Nesta abordagem educacional, os estudantes adquirem o conhecimento a partir de problemas interdisciplinares, desenhados para motivar o aprendizado e facilitar a transferência do conhecimento obtido. Sob a supervisão de um professor-tutor, os estudantes se organizam em pequenos grupos e discutem o problema, ativando conhecimentos prévios, gerando hipóteses explicativas e sínteses: a partir daí, se estabelecem os objetivos de aprendizagem. O estudo individual deve favorecer a resolução do problema de maneira colaborativa, englobando todas as etapas do Grupo Tutorial (GT). A boa elaboração do problema, a dinâmica do GT e as competências do professor-tutor são fatores essenciais para o sucesso da $\mathrm{ABP}^{5,6}$.

Ao se analisar a prática docente no curso, verifica-se no corpo de professores um alto nível de conhecimento técnico-científico, que se contrapõe às deficiências na formação didático-pedagógica e à heterogeneidade do corpo docente quanto à formação pedagógica e à resistência às mudanças, entre ou$\operatorname{tros}^{7}$. A opção metodológica do curso de Medicina da UEL, como proposto em seu projeto de curso, requer desses professores diferentes saberes, que evidenciam a complexidade da prática pedagógica, exigindo do docente mais do que o domínio do conteúdo. Ou seja, é essencial uma triangulação entre saberes da área lecionada, saberes da experiência profissional e saberes pedagógicos. Isto, conseqüentemente, traz conflitos, gera tensões, inquietações. E estas questões não devem ser desconsideradas ou naturalizadas, mas devem ser desenvolvidas estratégias de superação.
Este artigo apresenta os desafios enfrentados no processo de implementação de um projeto de educação permanente dos professores que atuam no curso de Medicina da Universidade Estadual de Londrina, com vistas ao desenvolvimento de uma prática pedagógica que leve em conta a realidade humana em toda a sua complexidade e diversidade.

\section{DESAFIOS E CONTEXTO DA EDUCAÇÃO PERMANENTE DOCENTE}

A formação profissional permanente dos docentes é primordial em programas que adotam inovações pedagógicas como referência educacional. Isto porque uma adequada formação e o comprometimento dos docentes oferecem suporte ao desenvolvimento do projeto pedagógico, cuja construção e execução são de responsabilidade da instituição, conforme a Lei de Diretrizes e Bases da Educação Nacional (LDBEN), n 9.394/967. Alguns autores ${ }^{8,9,10}$ mostram que a formação para a docência e o comprometimento dos docentes são elementos que dão suporte ao desenvolvimento do projeto educacional.

O movimento da educação permanente surgiu no início da década de 1970, como o momento mais importante da ruptura crítica do modelo escolar vigente. Segundo Canário ${ }^{2}$, a educação permanente tem caráter contínuo, aparece como um princípio reorganizador de todo o processo educativo, tem como ponto central a emergência da pessoa como sujeito da formação e se embasa em três pressupostos principais: a continuidade do processo educativo, sua diversidade e sua globalidade. Dessa forma, a educação permanente, assim concebida, enfatiza a dimensão cívica, indissociável da construção de uma cidade educativa.

Segundo Irby ${ }^{10}$, os componentes de uma educação permanente são: desenvolvimento instrucional que visa promover habilidades para o ensino, profissionalização docente, desenvolvimento de lideranças e desenvolvimento organizacional. Esses programas têm potencial para atingir aspectos relacionados ao desenvolvimento da carreira docente dos envolvidos, bem como suas relações intrapessoais e interpessoais ${ }^{11}$.

Nos programas de metodologias inovadoras, é necessário discutir coletivamente a ação docente, a integração dos conteúdos, a metodologia, as avaliações e os objetivos de estudo, com o propósito de questionar a melhor forma de o estudante apreender cada conteúdo, seja ele cognitivo, procedimental ou atitudinal ${ }^{12}$. O docente necessita mediar este processo para proporcionar a construção do domínio do conhecimento pelo discente, que deve participar ativamente numa ação intencional, deliberada e direcionada $a^{4,9,13}$.

Entre outros elementos, é também essencial saber trabalhar em grupo. Essa tarefa exige interação, respeito à singularidade, 
ética, habilidade para lidar com as emoções e flexibilidade. Esses são aspectos pedagógicos importantes e desafiantes na educação superior hoje. Na proposta de mudança, os docentes universitários necessitam de profissionalização na docência, numa perspectiva de ação colegiada, participativa e articulada ${ }^{4,9,13}$.

Venturelli ${ }^{13}$ refere que um bom profissional docente deve ter como competências os seguintes desempenhos:

- Facilitar aos estudantes a identificação de suas necessidades educacionais.

- Identificar o valor e as dificuldades do processo de ensino e intervir construtivamente para superá-las.

- Refletir sobre os objetivos, estratégias e orientações do programa e redimensioná-los quando necessário.

- Manejar as atividades que permitem estimular os estudantes para uma aprendizagem equilibrada e contextualizada em relação aos diferentes determinantes da saúde.

- Apoiar a conquista de um nível crítico, visando à autonomia profissional.

- Ser um modelo de avaliação, usando os princípios básicos de como fazer e receber críticas.

\section{ESTRATÉGIAS ADOTADAS}

A Comissão de Capacitação em Educação Médica (CCMED) do referido curso, ao longo dos dez anos de implantação do currículo integrado, vem desenvolvendo diversas atividades, instrumentos e mecanismos para a efetivação do mesmo ${ }^{14}$, em conjunto com outras comissões.

Uma das estratégias utilizadas tem sido a formação de professores-tutores. Foram realizados mais de 40 cursos de formação, e a CCMED desejava ampliar esse processo. Mas a pouca disponibilidade dos docentes e a "resistência" em discutir aspectos pedagógicos fizeram com que os momentos de encontro entre a Comissão e os professores sempre fossem muito mais informativos do que reflexivos ${ }^{14}$.

Com a contribuição de uma consultoria pedagógica em ensino superior para o curso, o grupo de professores que compõe a Comissão passou a refletir sobre suas atividades, tendo, inclusive, alterado o nome para Comissão de Educação Permanente em Educação Médica (CEPD-Med), considerando que a discussão da prática pedagógica deve ocorrer em todos os espaços, nos diversos cenários, em sala de aula, em reuniões de gestão do curso, e não somente em momentos pontuais, como cursos e palestras.

Como desdobramento desse processo de assessoria, a CEPD-Med incorporou algumas alterações no curso introdutório de formação de professores-tutores e incluiu em seu plano de ação para o biênio 2006-2007 uma estratégia de profissionalização docente. A princípio, pretendia-se que esta fosse a forma do curso de aperfeiçoamento, mas, por problemas institucionais, viabilizou-se como grupo de estudos.

\section{a) Formação de professores-tutores}

Anecessidade de continuar realizando os cursos de formação de professores-tutores é permanente, para oferecê-los aos novos docentes ou àqueles que ainda não o realizaram. Também é possível que a dinâmica do tutorial passe por evoluções naturais e venha a alterar alguns processos em certos momentos do curso (terceiro e quarto anos). Isso trouxe à Comissão o desafio de pensar uma nova proposta de curso, em que se pudessem iniciar as atividades de "exercício da função de professor-tutor" de maneira mais segura e confortável, por se ter experienciado "o ser estudante" num grupo tutorial.

Considerando a pouca disponibilidade de tempo dos docentes, a CEPD-Med vem propondo um projeto de curso de formação básica para professores-tutores desenvolvido em três etapas.

\section{Primeira etapa}

Com duração de quatro horas, consiste na apresentação do currículo integrado do curso de Medicina, incluindo objetivos e gestão, com ênfase na ABP e nos oito passos desta abordagem ${ }^{6}$ :

1. Leitura do problema - termos desconhecidos.

2. Definição do problema (formulação de questões).

3. Análise do problema com base em conhecimentos prévios (levantamento de hipóteses).

4. Resumo das conclusões.

5. Formulação de objetivos de estudo.

6. Auto-aprendizado.

7. Divisão dos conhecimentos com o grupo.

8. Avaliação formativa.

Em seguida, os participantes se dividem em pequenos grupos e realizam uma sessão tutorial, vivenciando o papel de estudantes, tutorados por um membro da Comissão. As dúvidas surgidas são discutidas, e, no final, cada participante recebe uma resenha com temas voltados para a educação de profissionais de saúde, publicadas na revista Educação em Poucas Palavras. Este material é retomado na terceira etapa sob a forma de apresentação oral pelos docentes participantes.

\section{Segunda etapa}

Com duração de seis horas, os futuros professores-tutores observam duas sessões tutoriais consecutivas (abertura e fecha- 
mento do problema) e preenchem um formulário com questões relacionadas à dinâmica tutorial e à vivência dos oito passos.

\section{Terceira etapa}

Com duração de quatro horas, inicia-se com a apresentação oral dos participantes das resenhas recebidas na primeira etapa. A seguir, é discutido o formulário preenchido pelos participantes da observação dos grupos tutoriais. Retomam-se as dificuldades mais freqüentes encontradas nas sessões tutoriais referidas pelos sujeitos envolvidos nessa atividade - docentes e discentes. Como atividade de síntese, faz-se uma análise comparativa do currículo integrado da UEL e do currículo grade vivido na formação de cada um dos participantes.

\section{b) Profissionalização docente}

Outra meta do programa de educação permanente da CEPDMed foi a constituição de um grupo de estudos para refletir sobre temas relacionados à prática pedagógica docente, com a assessoria de uma pedagoga com notório saber em educação superior.

Este grupo, denominado Grupo de Estudos Seqüenciais: Desafios do Ensino Médico, programou a realização de cinco encontros mensais e atividades presenciais e a distância, objetivando identificar os principais desafios presentes no ensino médico e analisando as ferramentas que a pedagogia universitária coloca à disposição dos docentes: a compreensão, renovação e valorização do papel docente no ensino e na aprendizagem, nos cursos de graduação. Os objetivos específicos estão agrupados nos seguintes temas: o processo grupal, a docência como profissão, os fundamentos legais, a organização curricular, e o ensinar e apreender.

Foram realizados dois encontros até o momento, com a participação de docentes do curso de Medicina e de outros cursos do Centro de Ciências da Saúde que adotam metodologias ativas. Possibilitou-se aos participantes uma reflexão sobre os elementos da historicidade da universidade determinantes de formas de gestão, da organização curricular e da atuação docente na universidade. Discutiu-se o papel da coordenação e do corpo docente do curso, visando integrar e facilitar aos professores a efetivação da docência como profissão.

Outro foco tratou da importância do conhecimento e aplicação dos fundamentos legais na constituição dos currículos universitários, em especial a LDBEN no 9.394-967 , das diretrizes curriculares e dos documentos institucionais delas derivados. Analisou-se a organização do currículo no curso de graduação em que atuam em relação às necessidades sociais atuais, possibili- dades e compromissos da universidade como instituição social, e às determinações legais.

Para os próximos encontros estão previstas a análise e a reflexão sobre o projeto político-pedagógico dos cursos e os avanços já efetivados ou ainda por se efetivarem na integração teoria-prática e básico-clínico, em busca da construção de uma visão de totalidade do curso. Pontua-se, nesta totalidade, que o todo em questão não resulta da simples soma, mas da integração articulada das partes. A matriz integrativa será tema de análise como ferramenta para facilitar a interdisciplinaridade dos conteúdos.

Os temas relacionados com o ensinar e apreender têm sido discutidos em todos os encontros como forma de pontuar a importância da ação conjunta e coletiva entre os docentes em direção da construção de princípios comuns aos cursos das diversas áreas. São focos: compreender o que é ciência, saber, saber ser, saber fazer e saber conviver, relacionando-os com os métodos de ensino e de pesquisa e com a organização dos conteúdos de ensino em cada fase do curso.

Ressalta-se, ainda, a discussão sobre o sujeito da aprendizagem, o estudante universitário, como um ser histórico, que vive processos cognitivos, com possibilidades e desafios em relação à aprendizagem para a construção de sua autonomia como futuro profissional. Além disso, pretende-se ampliar o conhecimento acerca do processo de acompanhamento, ou seja, a avaliação como processo.

Nesses encontros, tem-se utilizado a metodologia dialética, que tem como um dos princípios o sentido de que a visão inicial de cada um dos participantes pode ser sempre superada por um conhecimento mais complexo por meio de processos sistemáticos de análise. Para isso, utilizamos diversas estratégias, como discussões coordenadas no grupo geral e em subgrupos, apresentação de sínteses com vistas a reencaminhamentos, análise dos planos de ensino ou programas de aprendizagem, do processo de ensino e aprendizagem, e do processo de avaliação efetivados nos cursos de graduação em relação à atual organização curricular, visando a novas possibilidades integrativas.

\section{CONSIDERAÇÕES FINAIS}

O corpo docente é parte essencial do processo de implantação, sustentação e consolidação de mudanças curriculares, constituindo-se como agente central de transformação da realidade educacional. Propomos uma participação reflexiva, cooperativa, coletiva e integradora para superar conflitos e dificuldades desnecessários.

Quanto à formação inicial de professores-tutores, a percepção dos participantes foi de que a vivência de um GT permitiu des- 
mistificar a aplicação dos oito passos. Também possibilitou a troca de experiências e minimizou a angústia e as debilidades de quem está iniciando como professor-tutor. ACEPD-Med conclui que a proposta de curso foi positiva, mas é necessário criar mecanismos de educação permanente de habilidades de tutoria, para fortalecer o corpo docente.

De acordo com Venturelli13 ${ }^{13}$, a proteção do acervo docente implica estabelecer um programa de desenvolvimento acadêmico progressivo, para melhorar o desempenho docente na universidade, evitando sua frustração e o abandono da atividade acadêmica, e estimulando o estabelecimento de relações baseadas na troca e na confiança que dêem suporte a seu desenvolvimento. Isto porque o processo de mudança vem acompanhado de sentimentos de incerteza, o que leva a resistências pelo medo do desconhecido, por não se sentir parte da construção deste processo e também pela pouca profissionalização para a prática docente.

Com relação ao Grupo de Estudos Seqüenciais: Desafios do Ensino Médico, os organizadores mantém o acompanhamento dos participantes de forma processual. Isto acontece nos encontros presenciais e a distância, focando-se as produções individuais e coletivas nos grupos de estudo. Uma das expectativas do grupo organizador é que, ao término da programação, os participantes atuem como multiplicadores do conteúdo trabalhado e desencadeiem mudanças em seu loco de trabalho.

Neste contexto, alguns autores ${ }^{13,15,16}$ referem a importância de investir na profissionalização docente, criando redes colaborativas para garantir o aperfeiçoamento permanente do corpo docente, com a transformação de sua prática. Tem-se observado em diversos processos similares ${ }^{17-21}$ que os profissionais da área de saúde atuam com maior tranqüilidade e criatividade nestes contextos quando se tornam co-responsáveis pelo processo de transformação na consolidação do curso.

A superação dos desafios do processo de profissionalização do docente médico requer que se avaliem constantemente todas as iniciativas implantadas, pois este processo de acompanhamento auxiliará um aperfeiçoamento contínuo. Considerando o movimento que vivem os cursos da área médica no contexto nacional e internacional, trata-se, hoje, de uma necessidade premente.

\section{REFERÊNCIAS}

1. Walton H. World summit on medical education. Medic Educ 1994;28(Supl.1):142-49.

2. Canário R. A escola tem futuro? Das promessas às incertezas. Porto Alegre: ArtMed; 2006.
3. Santos BS. A universidade no século XXI. Para uma reforma democrática e emancipatoria da Universidade. 2. ed. São Paulo: Cortez; 2005.

4. Anastasiou LGC, Alves LP. Processos de ensinagem na universidade: pressupostos para as estratégias de trabalho em aula. 6. ed. Santa Catarina: Ed. UNIVILLE; 2006.

5. Schmidt HG. Educational Aspects of Problem-Based Learning. In: Jochems WMG, ed. Aktiverend onderwijs. Delft: Delftse Universitaire Pers; 1990.

6. Universidade Estadual de Londrina. Colegiado do curso medicina. Projeto político-pedagógico do curso de medicina - PPPMed. Londrina; 2004.

7. Brasil. Lei no. 9394, de 20 de dezembro de 1996. Estabelece as Diretrizes e Bases da Educação Nacional. [online]. [capturado 12 dez 2007]. Disponível em http:/ / www.planalto.gov.br/CCIVIL_03/LEIS/L9394.htm.

8. Wilkerson L, Irby DM. Strategies for improving teaching practices: a comprehensive approach to faculty development. Acad Med 1998;73(4):387-96.

9. Murray I, Savin-Baden M. Staff Development in Problem-based Learning. Teach High Educ 2000;5:107-26.

10. Irby DM. Models of faculty development for problem-based learning. Adv Health Sci Educ Theory Pract 1996;1:69-81.

11. Knight AM, Carrese JA, Wright SM. Qualitative assessment of the long-term impact of a faculty development programme in teaching skills. Med Educ 2007;41:592-600.

12. Zaballa A. A prática educativa: como ensinar. Porto Alegre: ArtMed; 1998.

13. Venturelli J, Fiorini VML. Programas educacionais inovadores em escolas médicas: capacitação docente. Rev Bras Educ Med 2001;25(3):7-21.

14. Almeida HGG, Ferreira Filho OF, Colus IMS, Linhares REC, Takahashi OC, Aguiar AC. Elaborando a capacitação de tutores: subsídeos para a prática. Rev Bras Educ Med 2003;27(3):191-99.

15. Moust JHC, VanBerkel HJM, Schmidt HG. Signs of erosion: reflections on three decades of problem-based learning at Maastricht University. Higher Education 2005;50:665-83.

16. Schuwirth LWT, Van Der Vleuten CPM. Challenges for educaionalists. BMJ 2006; 333:544-46.

17. Anastasiou LGC. Metodologia do ensino superior: da prática docente a uma possível teoria pedagógica. Curitiba: IBPEX; 1998.

18. Anastasiou LGC. Docência no ensino superior e os saberes científicos e pedagógicos. Revista Univille, Educação e Cultura jun 2002; 7(1). 
19. Anastasiou LGC. Desafios de um processo de profissionalização continuada: elementos da teoria e da prática. Revista Saberes, maio/ago 2000; 1.

20. Anastasiou LGC. Educação superior e preparação pedagógica: elementos para um começo de conversa. Revista Saberes, maio/ago 2001; 2(2).

21. Anastasiou LGC, Pimenta SG, Cavallet VJ. Docência no ensino superior: construindo caminhos. In: Severino AJ, Fazenda ICA, org. Formação docente: rupturas e possibilidades. Campinas, SP: Papirus; 2002.

\section{CONFLITOS DE INTERESSE}

Declarou não haver

\section{ENDEREÇO PARA CORRESPONDÊNCIA}

Maria José Sparça Salles de Faria

Rua Prefeito Hugo Cabral, 1065 - apto 501

86020-918 - Londrina - Paraná

maze@uel.br 